\title{
Revisiting the role of the pathological grading in pediatric adrenal cortical tumors: results from a national cohort study with pathological review
}

\author{
Cécile Picard $\mathbb{D}^{1} \cdot$ Daniel Orbach ${ }^{2} \cdot$ Matthieu Carton $\mathbb{D}^{3} \cdot$ Laurence Brugieres $^{4} \cdot$ Karine Renaudin $^{5}$. \\ Sébastien Aubert ${ }^{6} \cdot$ Dominique Berrebi $^{7} \cdot$ Louise Galmiche $^{8} \cdot$ Fanny Dujardin $^{9} \cdot$ Pierre Leblond $^{10}$. \\ Cécile Thomas-Teinturier ${ }^{11,12} \cdot$ Frédérique Dijoud $^{1}$
}

Received: 22 September 2018 / Revised: 21 October 2018 / Accepted: 22 October 2018 / Published online: 6 November 2018

(c) United States \& Canadian Academy of Pathology 2018

\begin{abstract}
The prognosis of malignant pediatric adrenocortical tumors is closely related to disease stage, which is used to guide perioperative treatment recommendations. However, current scoring systems are inadequate to distinguish between benign and malignant adrenocortical tumors. Robust microscopic prognostic features that could help determine perioperative therapy are also lacking. The aim of this national study was to review the prognostic value of the Wieneke scoring criteria and Ki67 labeling index in unselected pediatric adrenocortical tumors. Using strict definitions previously defined by expert pathologists, a Wieneke score was re-attributed to each tumor after an independent and centralized review. In addition, Ki67 proliferation index was performed and reviewed for each case. A total of 95 cases were selected; all were treated between 2000 and 2018 and had histopathologic material and sufficient outcome-related information available. Localized disease was found in $88 \%$ of patients. Among those with advanced disease, $6 \%$ had tumor extension into adjacent organs and 5\% had metastases at diagnosis. Median follow-up was 5 years and 3 months. The 5-year PFS was 82\%, 95\% CI [73\%-91\%]. Tumor stage significantly correlated with PFS $(p<0.0001)$. Tumor weight up to $200 \mathrm{~g}$, extra-adrenal extension and initial non-complete surgical resection were statistically associated with worse outcomes. No recurrences nor metastases occurred when the Ki67 index was $<15 \%$. Up to two of the following five factors including tumor necrosis, adrenal capsular invasion, venous invasion, mitotic count $>15 / 20$ high-power fields, and Ki67 index $>15 \%$, significantly correlated with worse outcomes. We propose a pathological scoring system incorporating the Ki67 index as part of a two-step approach after disease staging to guide adjuvant treatment in pediatric adrenocortical tumors, especially after incomplete resection. These results should be validated in an independent cohort.
\end{abstract}

Cécile Picard

cecile.picard@chu-lyon.fr

1 Department of Pathology, Groupement Hospitalier Est, Hospices Civils de Lyon, UCBL Lyon 1 University, Lyon, France

2 SIREDO Oncology Center, Institut Curie, PSL University, Paris, France

3 Unité de Biométrie, DRCI-Ensemble hospitalier, Institut Curie, 92210 Saint-Cloud, France

4 Département de Cancérologie de l'Enfant et l'Adolescent, Gustave Roussy Cancer Campus, Villejuif, France

5 Department of Pathology, Centre Hospitalier Universitaire de Nantes, Nantes, France

6 Department of Pathology, Centre de Biologie Pathologie, 59037 Lille Cedex, France
7 Department of Pathology, Hôpital Robert Debré, Assistance Publique-Hôpitaux de Paris, Paris, France

8 Department of Pathology, Hôpital Necker, Assistance PubliqueHôpitaux de Paris, Paris, France

9 Department of Pathology, Centre Hospitalier de Tours, Tours, France

10 Department of Pediatric Oncology, Centre Oscar Lambret, Lille, France

11 AP-HP, Hôpitaux Universitaires Paris-Sud, Site Bicêtre, Department of Pediatric Endocrinology, 94270 Le Kremlin-Bicêtre, France

12 INSERM UMR 1018, Cancer and Radiation Team, Institut Gustave Roussy, F-94805 Villejuif, France 


\section{Introduction}

Adrenocortical tumors in children are rare; they represent $0.2 \%$ of all pediatric malignancies and $5-6 \%$ of all adrenal tumors [1-3]. Their global incidence is $0.2-0.3$ new cases per one million children per year, but the incidence varies significantly across geographic regions depending on genetic background $[4,5]$. In France, 10 new cases are projected each year. Recent tremendous progress in the understanding of pathogenetic mechanisms that give rise to pediatric adrenocortical tumors have shown that these tumors have very specific clinical, histological, and molecular features distinguishing them from their adult counterparts [6]. Indeed, there is some evidence that pediatric adrenocortical tumors, especially those arising during the first 4 years of life, may be derived from fetal adrenal cells, which could explain their improved outcomes compared with tumors arising in adolescents or young adults [2,7].

The prognosis of adrenocortical tumors in children is highly dependent on the different tumor-related factors that define the disease's pathological staging, as first described by Sandrini et al., and now validated by the Children's Oncology Group [1, 2, 8, 9]. In addition, this staging is used to guide current treatment recommendations in the management of pediatric adrenocortical tumors. Apart from hormonal data, the four stages are determined by the following major prognostic factors: tumor weight and volume (with the use of $100 \mathrm{~g}$ and/or $200 \mathrm{~cm}$ [3] thresholds), initial extension of the disease, and quality of surgical resection (micro- or macroscopic residual disease). Pathological examination is crucial to document these major tumorrelated items, but the question remains regarding the prognostic value of isolated microscopic features in this context, especially for localized disease.

Whereas pathologists have reliable and reproducible scoring systems to characterize adult adrenocortical tumors (i.e., the well-known Weiss grading system), these diagnostic and prognostic criteria lack sensitivity and specificity to describe and predict pediatric adrenocortical tumors outcomes [9-13]. Indeed, pediatric tumors, which are classified as malignant according to adult pathological features, generally show better clinical outcomes than they would in adults. Therefore, the clear-cut line between benign adenoma and malignant adrenocortical carcinoma remains challenging to assess. To address this, Wieneke et al. developed a pediatric multi-parametric scoring system 15 years ago, which includes nine criteria and divide patients into three prognostic groups (benign long-term clinical outcome, indeterminate for malignancy, and poor clinical outcome) [12]. The nine criteria include four macroscopic features that relate to tumor dimension and extension (tumor weight $>400 \mathrm{~g}$, tumor size $>10.5 \mathrm{~cm}$, extension into periadrenal soft tissues and/or adjacent organs, invasion into vena cava), and five microscopic features derived from the adult Weiss scoring system (venous invasion, capsular invasion, presence of tumor necrosis, mitotic count $>15$ per 20 high-power fields, and presence of atypical mitotic figures). However, while this scoring system appeared to be prognostic in a few small series of patients, there is still controversy regarding its prognostic specificity, as some histologically malignant tumors (score $>3$ ) result in favorable clinical outcomes [12, 14-19]. Thus, additional prognostic factors have been assessed, with the Ki67 labeling index showing great promise in predicting patient outcomes for both adult and pediatric adrenocortical tumors [20,21].

The aim of this study was to collect a large number of unselected pediatric adrenocortical tumors from across the country, and to perform a national retrospective centralized pathological review of these cases by a group of experienced pathologists following the precise definition of Wieneke's microscopic features. Based on our review, we sought to determine the prognostic value of each item and to try and predict unfavorable pathological factors independent of clinical features.

\section{Materials and methods}

\section{Data and tumor collection}

This French retrospective multicenter study comprised all pediatric adrenocortical tumors diagnosed from January 2000 to January 2018 in pathology departments associated with 23 French University Hospitals. Cases were identified based on records of all confirmed benign, intermediate, and malignant primary adrenal neoplasms diagnosed in patients aged $0-18$ years old. Inclusion criteria were the availability of histopathologic material (at least paraffin blocks or unstained sections for histologic review), and sufficient demographic and outcome-related information. Among 113 cases of pediatric adrenocortical tumors that were recorded in pathology department databases, 95 were selected for the present study.

This study was approved by the Commission Nationale Informatique et Liberté (French Data Protection Authority, ${ }^{\circ} 2172992 \mathrm{v} 0$ on 18 August 2018). A letter of nonopposition to study participation was sent to living patients and their relatives, which describe the aims of the study and guaranteed that the patient's personal details would remain anonymous. Information collected from clinical files at each center included demographic details (age, gender, known or suspected cancer predisposition syndrome), initial clinical presentation (endocrine and non-endocrine symptoms, delay between symptom presentation and diagnosis, hormone values), tumor 
characteristics (size/volume of the primary tumor, extent of disease, genetic analysis performed), treatment modalities (for primary and recurrent or metastatic disease, if applicable), and outcomes (remission, relapse, diseasespecific mortality). The International Pediatric Adrenocortical Tumor Registry's (IPACTR) modified tumor staging system was used, which has been validated by the Children's Oncology Group (COG) [8], and is defined by: Stage I: Tumor completely resected with negative margins, weight $\leq 100 \mathrm{~g}$ or $\leq 200 \mathrm{~cm}^{3}$, no metastasis; Stage II: Tumor completely resected with negative margins, weight $>100 \mathrm{~g}$ or $>200 \mathrm{~cm}^{3}$, no metastasis; Stage III: Residual or inoperable tumor; Stage IV: Metastasis at presentation.

\section{Histology}

Two consensus review sessions among up to six expert pathologists in endocrine and/or pediatric pathology were held to precisely define the nine Wieneke histopathologic features associated with an increased probability of malignant clinical behavior [12]. The consensus definitions are as follows:

1. Tumor weight $>400 \mathrm{~g}$.

2. Tumor size $>10.5 \mathrm{~cm}$.

3. Extension into periadrenal soft tissues and/or adjacent organs.

4. Invasion into the vena cava.

5. Venous invasion - defined as unquestionable vascular venous emboli within the tumor capsule and independent of the main tumor proliferation. Tumor cuff protruding into the adrenal central vein was not considered venous invasion.

6. Capsular invasion - defined as tumor cells extending through the adrenal capsule, with unquestionable extension into periadrenal soft tissues. When the tumor was encapsulated, the extension beyond the tumor capsule with no extension through the adrenal capsule was not considered as a capsular invasion as the tumor stayed intra-adrenal.

7. Presence of tumor necrosis - defined as a confluent sheet of at least 10 necrotic cells. Calcifications or cholesterol cleft formation within the tumor were not considered as tumor necrosis.

8. Mitotic count - greater than 15 per 20 high-power fields, evaluated by counting 20 high-power fields ( $\times 400$ magnification) in at least five slides displaying the highest mitotic activity. More fields per slide were reviewed when less than five slides were available.

9. Presence of atypical mitotic figures - defined as abnormal chromosomal distribution or excess number of mitotic poles.
Using these strict definitions, hematoxylin and eosinstained slides were then centrally reviewed, separately and independently for each case by at least two pediatric pathologists, without knowledge of the patient's clinical outcome. We followed Wieneke's initial classification system, such that tumors with two or fewer criteria were classified as pathologically "benign", those with three as "indeterminate" for malignancy, and those with four or more criteria as "malignant" [12]. Additional microscopic features were assessed for each case including evidence of pre-operative tumor rupture, characterized by an inflammatory infiltrate with siderophages and foamy histiocytes extending beyond the adrenal capsule and into the periadrenal soft tissues.

\section{Immunohistochemistry}

Unstained glass slides were selected from a single representative block for each primary tumor for immunohistochemical analysis within a single center (Department of Pathology, Mother and Child University Hospital, Hospices Civils de Lyon). To assess Ki67 nuclear expression, $4 \mu \mathrm{m}$ thick, formalin-fixed, paraffin-embedded sections were stained using routine protocols with a commercially available antibody: mouse monoclonal MIB1 antibody (1:400 dilution; Dako, Glostrup, Denmark) against Ki67. The immunostains were scanned at $\times 400$ magnification and automatically digitized in an appropriate file format. Hot spot areas within each tumor were selected based on visual estimation, and static images of these proliferative areas were printed. To assess the Ki67 labeling index, a formal manual count of Ki67 positively stained nuclei was performed on at least 2000 tumor cells by two independent observers for each case.

\section{Statistical analysis}

All data were analyzed using descriptive statistical methods, and the proportion of patients within each group of characteristics were compared using the chi-squared or Fisher's exact test, when appropriate. A $p$-value of $<0.05$ was considered statistically significant. Survival time was calculated from the date of diagnosis to the time of last follow-up or event. Non-remission, tumor progression or relapse, and death by any cause were considered for progression-free survival (PFS). Overall survival (OS) was measured from the date of diagnosis to death for any reason. Both OS and PFS were estimated using the Kaplan-Meier method, and median follow-up duration using the reverse Kaplan-Meier method. Survival curves with their log-rank tests were generated. To evaluate interobserver agreement between the microscopic criteria described in the initial pathology report 
Table 1 Clinical features and data on therapy strategy for the whole cohort

\begin{tabular}{|c|c|c|c|}
\hline Feature & Value & Missing data & $\%$ \\
\hline \multicolumn{4}{|l|}{ Gender } \\
\hline Female & 67 & - & 70 \\
\hline Male & 28 & - & 30 \\
\hline \multicolumn{4}{|l|}{ Age (year) } \\
\hline$<4$ & 43 & - & 45 \\
\hline $4-10$ & 25 & - & 26 \\
\hline$>10$ & 27 & - & 28 \\
\hline \multicolumn{4}{|l|}{ Clinical presentation $^{\mathrm{a}}$} \\
\hline Virilization & 55 & 9 & 64 \\
\hline Cushing syndrome & 22 & 11 & 26 \\
\hline Hypertension & 19 & 11 & 23 \\
\hline Palpation of a mass & 11 & 11 & 13 \\
\hline No clinical symptom & 3 & 11 & 4 \\
\hline Functional tumor & 74 & 11 & 88 \\
\hline \multicolumn{4}{|l|}{ Tumor diameter (by imaging) } \\
\hline $2-5 \mathrm{~cm}$ & 40 & & 43 \\
\hline $5-10 \mathrm{~cm}$ & 36 & & 39 \\
\hline$>10 \mathrm{~cm}$ & 16 & & 17 \\
\hline Data not specified & & 3 & \\
\hline \multicolumn{4}{|l|}{ Stage (Children's Oncology Group staging) } \\
\hline I & 54 & & 58 \\
\hline II & 16 & & 17 \\
\hline III & 18 & & 19 \\
\hline IV & 5 & & 5 \\
\hline Data not specified & & 2 & \\
\hline Initial percutaneous biopsy & 9 & 6 & 10 \\
\hline Initial surgery & 86 & & 90 \\
\hline \multicolumn{4}{|l|}{ Results of initial surgery } \\
\hline No residual tumour & 71 & & 83 \\
\hline $\begin{array}{l}\text { Micro- or macroscopic residual tumour } \\
\text { (including tumor rupture) }\end{array}$ & 14 & & 16 \\
\hline Data not specified & & 1 & \\
\hline No initial surgery & 7 & & 7 \\
\hline Biopsy only & 2 & & 2 \\
\hline $\begin{array}{l}\text { Delayed surgery (after pre-operative } \\
\text { therapy) }\end{array}$ & 5 & & 5 \\
\hline Data non-specified on surgery modalities & & 2 & \\
\hline \multicolumn{4}{|l|}{ Medical therapies } \\
\hline Mitotane & 20 & & 22 \\
\hline Neoadjuvant & 4 & & \\
\hline Adjuvant & 13 & & \\
\hline Both & 3 & & \\
\hline Data not specified & & 5 & \\
\hline Conventional chemotherapy regimens & 13 & & 15 \\
\hline Neoadjuvant & 4 & & \\
\hline Adjuvant & 8 & & \\
\hline Both & 1 & & \\
\hline
\end{tabular}

Table 1 (continued)

\begin{tabular}{llll}
\hline Feature & Value & Missing data & $\%$ \\
\hline Data not specified & & 6 & \\
Radiation therapy & 1 & 8 & 1 \\
Initial & 0 & & \\
Palliative & 1 &
\end{tabular}

${ }^{\mathrm{a}}$ Multiple presentations possible

and those attributed to the tumor after our review, the kappa statistic was performed. Originally described by Cohen, the higher the kappa value, the better the agreement [22]. A kappa value $>0.80$ indicates excellent agreement beyond chance; those between 0.61 and 0.80 good agreement beyond chance; those between 0.41 and 0.60 moderate agreement beyond chance; and values $<0.40$ indicate poor agreement beyond chance. For multivariate analyses, a Cox regression analysis using Firth's penalized likelihood method was used to estimate hazard ratios (HRs), as certain variables had no events and/or deaths [23].

\section{Results}

\section{Clinical and treatment characteristics}

Overall, 95 patients were selected (Table 1). The median age at diagnosis was 5 years (range: $0-17$ ). A cancer predisposition genetic syndrome was known in 24 cases, and the genetic status was unknown in 18 cases. Functional tumors accounted for 74 patients (88\%), with androgen excess being the most common (60 cases, 75\%). Based on imaging, 82 patients had localized disease (88\%), 6 displayed extension into adjacent organs (7\%), and 5 had metastases (5\%). According to Children's Oncology Group staging, 54 had stage I disease (58\%), 16 stage II (17\%), 18 stage III (19\%), and 5 stage IV (5\%). Data on initial disease extension were missing for two patients.

An initial diagnostic percutaneous needle biopsy was performed for nine patients. Of these, seven underwent a subsequent surgical resection of their tumor. The remaining two patients did not have definitive surgery because of locally advanced disease. Initial treatment modalities are summarized in Table 1. Tumor spillage during initial surgery occurred in four cases of primary surgery. An initial complete remission occurred in 76 patients $(84 \%$, missing data for five patients).

\section{Outcomes}

After a median follow-up of 5 years and 3 months (min: 2 months; max: 17 years), 13 patients relapsed, with distant 
Table 2 Pathologic findings after review

\begin{tabular}{|c|c|c|c|}
\hline Feature & Value & Missing data & $\%$ \\
\hline \multicolumn{4}{|l|}{ Gross findings } \\
\hline Tumor location & & 2 & \\
\hline Right adrenal & 45 & & 48 \\
\hline Left adrenal & 48 & & 52 \\
\hline Tumor weight, $g$ & & 7 & \\
\hline Median (min-max) & $68(2.9-1870.0)$ & & \\
\hline Tumor size, $\mathrm{cm}$ & & 5 & \\
\hline Median (min-max) & $6(2-22)$ & & \\
\hline Tumor extension & & 2 & \\
\hline $\begin{array}{l}\text { Extension into periadrenal } \\
\text { soft tissues }\end{array}$ & 12 & & 13 \\
\hline $\begin{array}{l}\text { Extension into adjacent } \\
\text { organ }\end{array}$ & 2 & & 2 \\
\hline Vena cava invasion & 6 & 6 & 7 \\
\hline \multicolumn{4}{|l|}{ Microscopic findings } \\
\hline Vascular invasion & 24 & 4 & 26 \\
\hline Capsular invasion & 22 & 5 & 24 \\
\hline Mitotic count $>15 / 20 \mathrm{HPF}$ & 21 & 2 & 23 \\
\hline Atypical mitotic figures & 55 & 2 & 59 \\
\hline Necrosis & 49 & - & 52 \\
\hline Pre-operative rupture changes & 10 & 14 & 12 \\
\hline \multicolumn{4}{|l|}{ Wieneke score ${ }^{\mathrm{a}}$} \\
\hline$<3$ & 60 & & 63 \\
\hline$=3$ & 11 & & 12 \\
\hline$>3$ & 24 & & 25 \\
\hline
\end{tabular}

$H P F$ high-power fields

${ }^{a}$ Missing Wieneke criterion were considered to be equal to 0 in order to estimate definitive Wieneke score

metastases in 12 cases. Among those patients with relapsed disease, eight had initially localized disease (six stage III, and two stage II). Death was associated with disease progression in 11 cases including 9 with rapid evolution without complete remission. Five patients died as a result of causes unrelated to tumor progression such as drug toxicity $(n=3)$, progression of metachronous tumor $(n=1)$, or other (traffic accident, $n=1$ ).

The 2-year and 5-year PFS of the overall study population were 85 (95\% confidence interval (CI) [78-93\%]) and $82 \%$ (95\% CI [73-91\%]), respectively. The 2-year and 5 -year OS were $86 \%$ for both (95\% CI [78-93\%]).

\section{Pathological and immunohistochemical findings}

Gross pathological findings are described in Table 2. The majority of tumors weighed $<100 \mathrm{~g}(n=54,63 \%)$. Tumor weight up to $200 \mathrm{~g}$ statistically affected OS and PFS (HR: 9.32, 95\% CI [2.99-29.0], $p<0.001$ and HR: $16.4,95 \%$ CI [5.51-48.7], $p<0.001$, respectively). Three patients had radiographically confirmed lymph node involvement, and two of these patients were confirmed histologically. The remaining patient did not undergo surgery due to rapidly fatal disease progression.

A Wieneke score was given in the original pathology report for 42/95 cases. A Weiss score (i.e., adult score) was specified in 60/95 cases. Microscopic pathological findings obtained after our pathological review are summarized in Table 2. The Ki67 labeling index was $\geq 15 \%$ in 37 of 87 patients with analyzable tumor samples.

\section{Interobserver agreement in the assessment of the Wieneke score}

The interobserver agreement for each microscopic Wieneke criteria between the initial pathology report and our centralized review was assessed for 89 cases. A strong concordance was seen for confluent necrosis assessment and venous invasion (kappa $=0.91$ and 0.81 , respectively), moderate for mitotic count $>15 / 20$ high-power fields and atypical mitotic figures (kappa $=0.72$ and 0.76, respectively), and poor for capsular invasion assessment $(k a p p a=0.54)$. The concordance was moderate for the overall assessment of the Wieneke score (kappa $=0.73)$.

\section{Prognostic factors (univariate analysis)}

Table 3 summarizes the survival probability according to risk factors at univariate analyses for clinical and tumor characteristics. Age at diagnosis appeared to be related to PFS and OS in comparison with gender and functional symptoms. Symptoms related to tumor volume, such as presence of a palpable mass, were associated with unfavorable outcomes. No deaths and no events occurred when patients were initially asymptomatic. All patients with regional lymph node involvement and/or metastasis died within 2 years of diagnosis $(p<0.0001$ for both). Initial biopsy and residual tumor after surgical resection (including tumor rupture) were statistically associated with worse outcomes. Higher tumor stage according to the Children's Oncology Group staging system significantly correlated with worse OS and PFS $(p<0.0001)$ (Fig. 1). No deaths occurred among patients with stage I disease.

Among the macroscopic pathological features, tumor weight and size and extension into periadrenal soft tissues and/or adjacent organs, but not vena cava invasion, were related to OS and PFS (Table 4). A univariate Cox regression analysis was performed for each microscopic Wieneke criterion attributed after our review (Table 4). Of the five microscopic Wieneke criteria, venous invasion, adrenal capsular invasion, mitotic count $>15 / 20$ high-power fields, and necrosis statistically affected OS and PFS (Fig. 2). No deaths and no events occurred when the tumor had no necrosis. Among the 10 cases with microscopic features 
Table 3 Outcome according to patient's and tumor's characteristics (univariate analysis)

\begin{tabular}{|c|c|c|c|c|c|c|}
\hline \multirow[b]{2}{*}{ Characteristic $^{\mathrm{a}}$} & \multicolumn{3}{|c|}{ PFS } & \multicolumn{3}{|l|}{ OS } \\
\hline & $n$ & HR $(95 \% \mathrm{CI})$ & $p$-Value & $n$ & HR $(95 \% \mathrm{CI})$ & $p$-Value \\
\hline \multicolumn{7}{|l|}{ Gender } \\
\hline Female & 67 & 1 & 0.648 & 67 & 1 & 0.544 \\
\hline Male & 28 & $0.77[0.25 ; 2.38]$ & & 28 & $0.67[0.19 ; 2.43]$ & \\
\hline \multicolumn{7}{|l|}{ Age at diagnosis (years) } \\
\hline$[0-4]$ & 43 & 1 & & 43 & 1 & \\
\hline$[4-10]$ & 25 & $0.94[0.17 ; 5.14]$ & 0.002 & 25 & $0.99[0.18 ; 5.41]$ & 0.002 \\
\hline$[10-17]$ & 27 & $5.95[1.85 ; 19.1]$ & & 27 & $4.55[1.37 ; 15.2]$ & \\
\hline \multicolumn{7}{|l|}{ Virilization } \\
\hline No & 31 & 1 & & 31 & 1 & \\
\hline Yes & 55 & $0.46[0.17 ; 1.28]$ & 0.139 & 55 & $0.43[0.14 ; 1.29]$ & 0.132 \\
\hline \multicolumn{7}{|l|}{ Cushing syndrome } \\
\hline No & 62 & 1 & & 62 & 1 & \\
\hline Yes & 22 & $0.71[0.20 ; 2.52]$ & 0.599 & 22 & $0.55[0.12 ; 2.49]$ & 0.438 \\
\hline \multicolumn{7}{|l|}{ Presence of a hypertension } \\
\hline No & 65 & 1 & 0.548 & 65 & 1 & 0.286 \\
\hline Yes & 19 & $1.42[0.45 ; 4.46]$ & & 19 & $1.90[0.58 ; 6.21]$ & \\
\hline \multicolumn{7}{|l|}{ Palpation of a mass } \\
\hline No & 73 & 1 & & 73 & 1 & \\
\hline Yes & 11 & $3.16[1.00 ; 9.94]$ & 0.051 & 11 & $4.89[1.60 ; 15.0]$ & 0.005 \\
\hline \multicolumn{7}{|l|}{ Functional tumor } \\
\hline No & 10 & 1 & & 10 & 1 & \\
\hline Yes & 74 & $0.47[0.13 ; 1.70]$ & 0.250 & 74 & $0.43[0.12 ; 1.58]$ & 0.202 \\
\hline \multicolumn{7}{|l|}{ Regional lymph node involvement } \\
\hline No & 87 & 1 & & 87 & 1 & \\
\hline Yes & 3 & $17.4[4.52 ; 67.5]$ & $<0.001$ & 3 & $27.6[5.98 ; 127]$ & $<0.001$ \\
\hline \multicolumn{7}{|l|}{ Presence of metastases } \\
\hline No & 88 & 1 & & 88 & 1 & \\
\hline Yes & 5 & $21.9[6.57 ; 73.3]$ & $<0.001$ & 5 & $21.1[6.53 ; 68.3]$ & $<0.001$ \\
\hline \multicolumn{7}{|l|}{ Initial biopsy } \\
\hline No & 80 & 1 & & 80 & 1 & \\
\hline Yes & 9 & $19.0[6.23 ; 58.0]$ & $<0.001$ & 9 & $15.9[5.17 ; 48.7]$ & $<0.001$ \\
\hline \multicolumn{7}{|l|}{ Initial surgery } \\
\hline No residual tumor & 71 & 1 & $<0,001$ & 71 & 1 & $<0.001$ \\
\hline Micro- or macroscopic residual tumor (including spillage) & 14 & $14.5[4.22 ; 49.6]$ & & 14 & $20.6[4.15 ; 102]$ & \\
\hline
\end{tabular}

$H R$ hazard ratio, $n$ number of patients, $P F S$ progression-free survival, $O S$ overall survival

${ }^{a}$ Sum does not add up to the total because of some missing values

suggesting pre-operative rupture, all but two cases had a Wieneke score $>3$ (Fig. 3). According to the Wieneke scoring system, cases with a score of 3 ("indeterminate" for malignancy, $n=11$ ) and $>3$ ("malignant", $n=24$ ), were associated with malignant clinical behavior (Table 4). Of the 24 patients with a score $>3$ included in this study, 11 patients died of their disease, 2 were alive with their disease, and 10 were in complete remission at the end of follow-up (1 patient with missing data).
OS and PFS correlated with Ki67. PFS was $100 \%$ for patients with tumors associated with a Ki67 index $<15 \%$ ( $p$ $<0.0001$ ) (Fig. 4).

A specific five-item microscopic score was created by selecting the most predictive microscopic criteria (using our refined consensus definitions), namely vascular invasion, adrenal capsular invasion, confluent necrosis, mitotic count $>15 / 20$ high-power fields and $\mathrm{Ki} 67 \geq 15 \%$. A score $>2$ was significantly associated with worse OS and PFS, regardless 


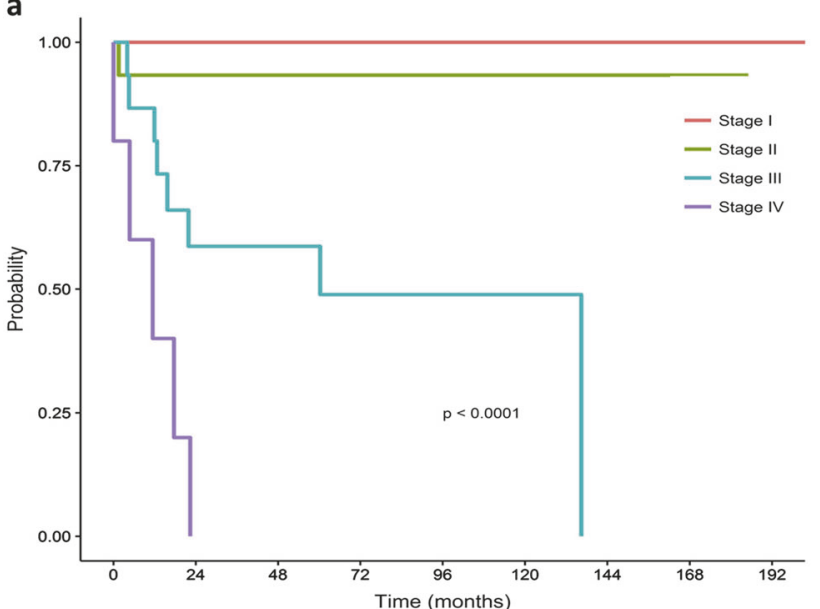

Number at risk

\begin{tabular}{|c|c|c|c|c|c|c|c|c|}
\hline$=$ & 53 & 41 & 30 & 20 & 13 & 9 & 6 & 2 \\
\hline$=$ & 15 & 13 & 10 & 4 & 4 & 3 & 3 & 2 \\
\hline & 15 & 8 & 7 & 5 & 3 & 1 & 0 & 0 \\
\hline & t & 0 & 0 & 0 & 0 & 0 & 0 & 0 \\
\hline
\end{tabular}

Fig. 1 Estimated overall survival (OS) (a) and progression-free survival (PFS) (b) for the whole cohort of patients with regards to tumor

of stage $(p<0.0001)$ (Fig. 5). No deaths occurred when the score was $<2$, regardless of stage. The subgroup analysis for stage II $(n=16)$ and stage III tumors $(n=18)$ showed no significant differences between patients with a score $\leq 2$ and patients with a score $>2$ regarding OS and PFS $(p=$ 0.16 and $p=0.015$, respectively, for stage II tumors, and $p=0.12$ and $p=0.27$, respectively, for stage III tumors).

\section{Prognostic factors (multivariate analysis)}

Significant factors from our univariate analyses including disease stage (Children's Oncology Group), non-functional symptoms or symptoms related to tumor volume (abdominal mass), age at diagnosis, tumor weight and the five-item microscopic score, were included in a multivariate Cox regression analysis using Firth's penalized likelihood method. Disease stage was the sole clinical factor that remained independently associated with PFS (HR 18.0 [0.6; 3215] for stage II and HR 64.7 [3.2; 9690] for stages III-IV, $p=0.045$ ). The five-item score was independently associated with prognosis: HR 15.4 [1.3597], $p=0.02$ for PFS and HR 18.1 [1.34708], $p=0.025$ for OS, for a score $>2$ versus $\leq 2$.

\section{Discussion}

Based on this large and comprehensive series of pediatric adrenocortical tumors, we propose a novel prognostic scoring system using pathological features available after

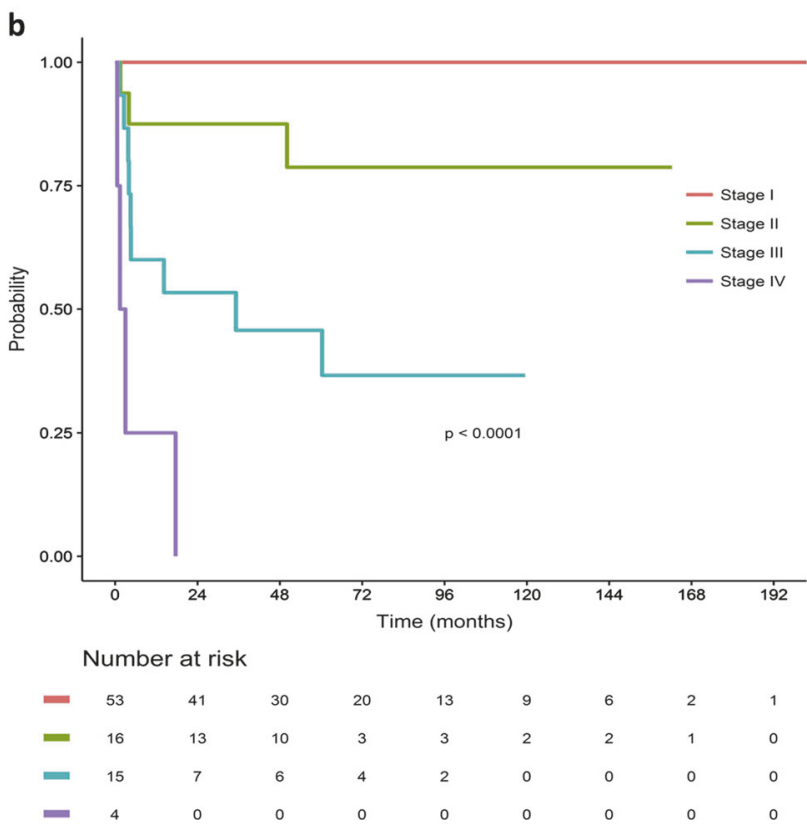

staging (as defined by the International Pediatric Adrenocortical Tumor Registry)

biopsy or initial surgery, regardless of tumor extensions. With only 95 cases over a 17 -year period, we confirmed the low incidence of these tumors (i.e., five to six cases each year among a population of 60-65 million inhabitants in France). In this setting, pathologists, even those specialized in pediatric pathology, usually do not have the opportunity to examine enough cases to gain solid expertise. Most pathologists acknowledge that some morphological criteria present in the different scoring systems, especially in the Wieneke score, remain poorly defined and observer dependent. This explains the heterogeneous use of this score between pathology departments, illustrated by our finding that less than half of the original pathology reports in this study mentioned the Wieneke score. Consequently, the first part of our study was dedicated to defining precise and clear-cut definitions of the Wieneke criteria to gain reproducibility and diagnostic reliability for the subsequent review of all included cases.

Tumor volume is a major factor that correlates with clinical outcomes in pediatric adrenocortical tumors, and in fact is described as the only predictor of malignancy by some authors $[16,24]$. The mean tumor weight in our series of unselected lesions was $193 \mathrm{~g}$, which appears to be low compared with other published pathological review series, in which the reported mean tumor weights were between 350 and $400 \mathrm{~g}[12,14,15,19]$. Depending on the sample size and inclusion criteria, the range of sizes and weights can vary greatly. For example, size and weight cutoffs used by Wieneke et al. ( $10.5 \mathrm{~cm}$ and $400 \mathrm{~g}$, respectively) were defined 15 years ago from a series of relatively advanced 
Table 4 Outcomes according to pathologic criteria after review (univariate analysis)

\begin{tabular}{|c|c|c|c|c|c|c|}
\hline \multirow[b]{2}{*}{ Criteria $^{a}$} & \multicolumn{3}{|c|}{ PFS } & \multicolumn{3}{|l|}{ OS } \\
\hline & $n$ & HR $(95 \% \mathrm{CI})$ & $p$-Value & $n$ & HR $(95 \% \mathrm{CI})$ & $p$-Value \\
\hline \multicolumn{7}{|l|}{ Weight $(\mathrm{g})$} \\
\hline$[2.9-100.0]$ & 54 & 1 & & 54 & 1 & \\
\hline$[100.0-1870.0]$ & 34 & $31.0[4.09 ; 235.3]$ & $<0.001$ & 34 & $24.4[3.17 ; 188]$ & 0.002 \\
\hline \multicolumn{7}{|l|}{ Size $(\mathrm{cm})$} \\
\hline$[2.0-10.5]$ & 73 & 1 & & 73 & 1 & \\
\hline$[10.5-22.0]$ & 17 & $13.3[4.66 ; 37.7]$ & $<0.001$ & 17 & $10.5[3.37 ; 32.8]$ & $<0.001$ \\
\hline Extension into periadrenal soft tissues & 12 & $9.75[3.56 ; 26.7]$ & $<0.001$ & 12 & $15.5[4.80 ; 50.3]$ & $<0.001$ \\
\hline Extension into adjacent organs & 2 & $31.9[5.25 ; 193]$ & $<0.001$ & 2 & $17.6[3.73 ; 83.6]$ & $<0.001$ \\
\hline Vena cava invasion & 6 & $2.78[0.63 ; 12.3]$ & 0.179 & 6 & $3.87[0.83 ; 18.0]$ & 0.084 \\
\hline Vascular invasion & 24 & $5.62[1.84 ; 17.2]$ & 0.003 & 24 & $4.93[1.38 ; 17.5]$ & 0,014 \\
\hline Capsular invasion & 22 & $6.47[2.11 ; 19.8]$ & 0.001 & 22 & $15.7[3.34 ; 74.3]$ & $<0.001$ \\
\hline Mitotic count $>15 / 20 \mathrm{HPF}$ & 21 & $3.26[1.21 ; 8.76]$ & 0.019 & 21 & $3.41[1.14 ; 10.2]$ & 0.028 \\
\hline Atypical mitotic figures & 55 & $3.16[0.89 ; 11.2]$ & 0.075 & 55 & $3.98[0.87 ; 18.2]$ & 0.075 \\
\hline Pre-operative rupture changes & 10 & $4.61[1.35 ; 15.8]$ & 0.015 & 10 & $3.38[0.84 ; 13.7]$ & 0.087 \\
\hline \multicolumn{7}{|l|}{ Wieneke score } \\
\hline$<3(n=60)$ & & 1 & & & 1 & \\
\hline$=3(n=11)$ & & $22.7[2.54 ; 203]$ & 0.002 & & $8.28[1.38 ; 49.6]$ & 0.003 \\
\hline$>3(n=24)$ & & $41.3[5.32 ; 320]$ & & & $14.6[3.15 ; 68.4]$ & \\
\hline
\end{tabular}

$H P F$ high-power fields, $H R$ hazard ratio, $n$ number of patients, $P F S$ progression-free survival, $O S$ overall survival

aThe sum does not add up to the total because of missing values. HR for the microscopic criteria "necrosis" were not calculable as there was no event when the tumor was devoid of necrosis diseases. These cutoffs are obviously statistically significant in our series but are likely too high to be discriminant as a weight cutoff of $100 \mathrm{~g}$ shows already strong prognostic value. Consistent with critical cutoffs used for tumor staging in localized disease and with previous risk group analyses, a tumor weight of up to $200 \mathrm{~g}$ appears to convincingly predict worse outcomes $[1,8,9,16]$. Furthermore, because weight varies proportionally with size, considering these two items together in the same scoring system for prognosis prediction likely overestimates malignancy.

In addition, extension into soft tissues and/or adjacent organs was confirmed to be a strong predictor of malignancy in our series, correlating with higher tumor volumes (median weight $327 \mathrm{~g}$ and median size $11 \mathrm{~cm}$ for tumors with regional extension vs. $52 \mathrm{~g}$ and $6 \mathrm{~cm}$ without). Notably, extension into adjacent organs was highly suspected by imaging before surgical resection in the two cases displaying this feature. Regarding tumor extension into soft tissues, this naturally implies capsular invasion (a second Wieneke criterion), resulting in the automatic assignment of two points to such tumors. It would perhaps seem more accurate to strictly define capsular invasion (with tumor extension through the adrenal capsule), which would then be sufficient to describe the microscopic invasion of surrounding tissues and periadrenal extension. Indeed, the use of this criterion was validated in our study by its strong reliability to predict outcomes and its improvement in interobserver reliability in the expert review compared with initial reports.

Vena cava invasion is also of great significance, but is mired by confounding factors since it generally corresponds to locally advanced tumors. Furthermore, this tumor characteristic is chiefly an imaging feature, which can be challenging to assess by the pathologist on a surgical resection specimen. This feature greatly influences the integrity of the surgical resection due to the increased risk of tumor emboli and pre- and perioperative rupture. In our series, its prognostic value was not significant, and it impacted OS only in the case of initial non-complete surgical resection (data not shown). Thus, the relevance of this criterion in the histopathologic score seems equivocal and does not provide any further information compared with disease staging.

Microscopically, architectural and cytologic findings are quite variable between neoplasms, but also from one region to another within the same lesion. As previously reported, most tumors are composed of sheets of cells with abundant eosinophilic cytoplasm and varying degrees of nuclear pleomorphism and atypia, and no one morphologic characteristic appears predictive of malignancy 16]. Among the Wieneke criteria, necrosis, along with capsular invasion, are the most consistent features of malignancy, followed by venous invasion and mitotic count $>15 / 20$ high-power 
a
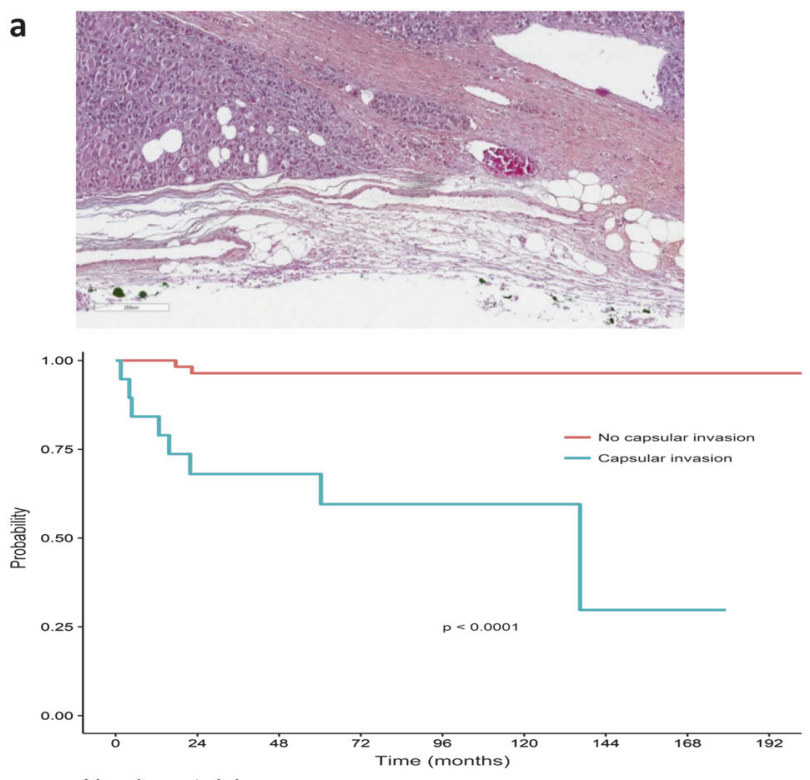

Number at risk

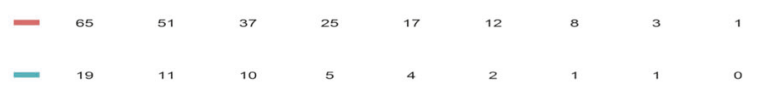

c
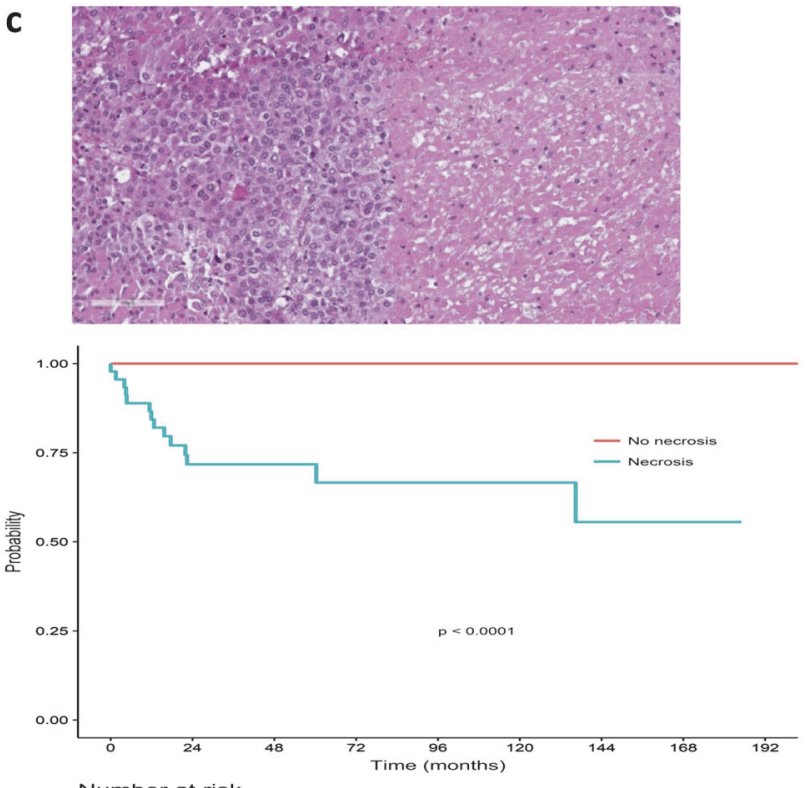

Number at risk

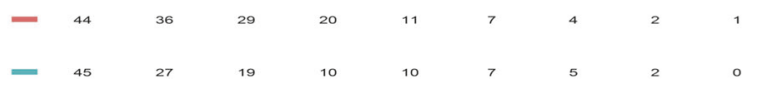

b
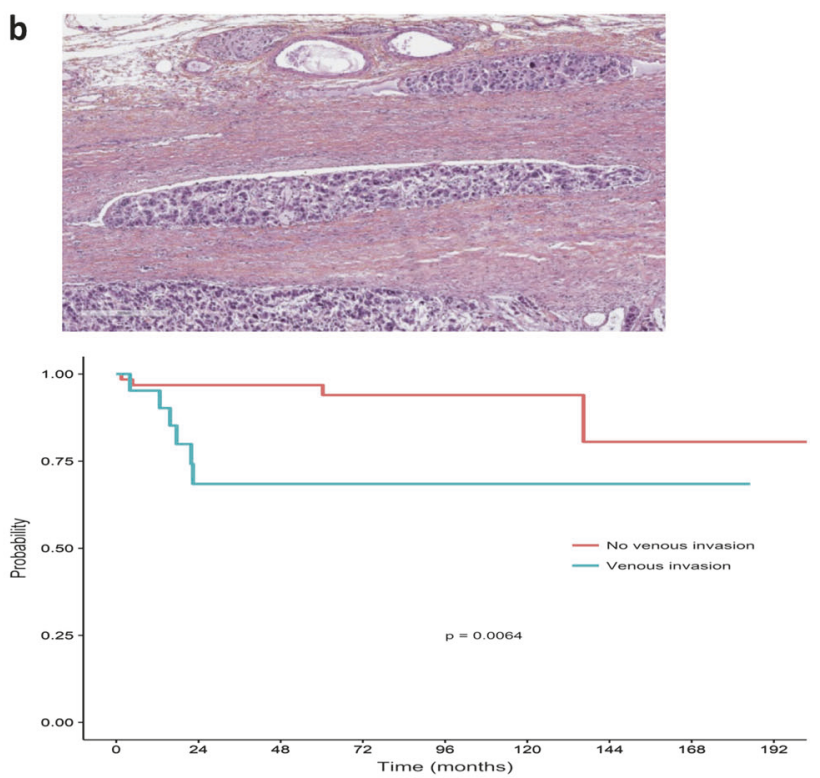

Number at risk

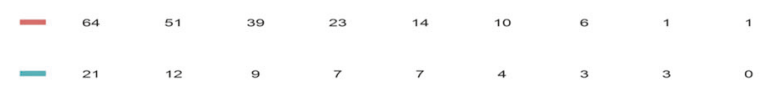

d
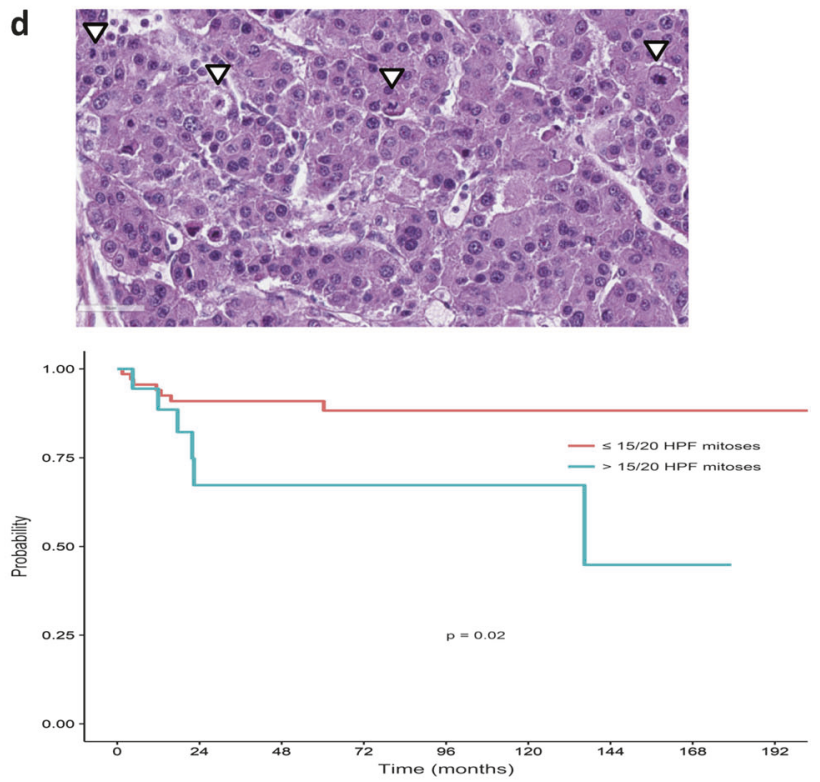

Number at risk

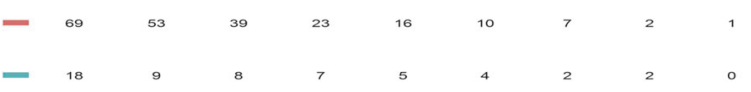

Fig. 2 Illustration of prognostic microscopic features attributed to the tumor after centralized review (hematoxylin and eosin, H\&E) and corresponding estimated overall survival (OS) for the whole cohort: a adrenal capsular invasion, b venous invasion, $\mathbf{c}$ confluent necrosis, and $\mathbf{d}$ mitotic count $>15 / 20$ high-power fields

fields. In our experience, necrosis was not specifically associated with malignancy because it was present in $30 \%$ of cases with a benign outcome. Nevertheless, large necrotic areas are present in the vast majority of histologically malignant tumors as reported in several different series, including ours $[12,14,15,19]$. Interestingly, no events and no deaths occurred when tumors were devoid of necrosis. The proportion of tumors displaying atypical mitotic figures is heterogeneous in various series including ours, present in both benign and malignant tumors [14, 19]. This likely reflects the difficulty that pathologists encounter in formally defining atypical mitotic figures, resulting in the poor interobserver reproducibility we observed in our study. 
Fig. 3 Microscopic changes in pediatric adrenocortical tumor evocative of pre-operative rupture (hematoxylin and eosin stains). a Fibro-inflammatory infiltrate that extends into periadrenal soft tissue at periphery of a tumor, $\times 40$. b This neocapsule is composed of mononuclear inflammatory cells, foamy histiocytes, and siderophages, $\times 100$
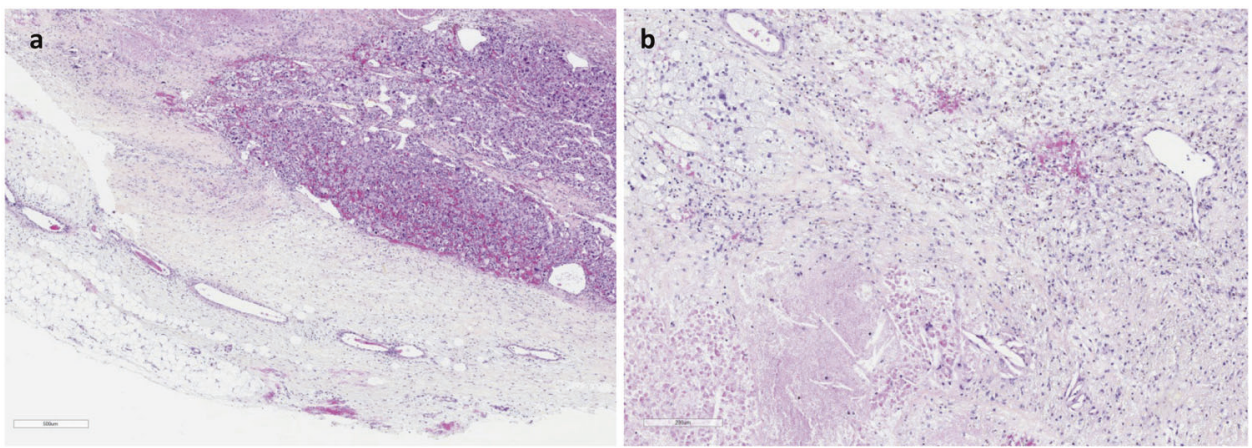

By reviewing a large number of tumors, we were able to note the presence of certain microscopic changes that were consistent with pre-operative rupture. This likely highlights the aggressive and proliferative potential of the tumor with iterative ruptures (inducing abdominal pain) and reformation of a tumor fibro-inflammatory neocapsule. Only a few tumors displayed this feature in our series, but when present, it showed a significant impact on 2-year PFS (91\% without microscopic rupture vs. $67 \%$ with rupture). This could be a supplementary criterion to classify the tumor as stage III disease (as defined by Children's Oncology Group) when there is no other evidence of residual tumor. It is fundamental to determine the rupture site, as retroperitoneal or intraperitoneal ruptures would likely have different clinical implications.

The Wieneke score in its entirety was predictive of patient outcomes when scored as "benign" $(<3)$, as no events occurred in this group of patients. OS in the "intermediate" Wieneke (score $=3$ ) subgroup was close to the "malignant" Wieneke subgroup. However, while most patients in the "malignant" subgroup indeed had unfavorable outcomes, a significant proportion had benign outcomes, highlighting the inability of the Wieneke score to predict malignancy with strong reliability.

No single morphologic feature in pediatric adrenocortical tumors is enough to differentiate benign and potentially malignant neoplasms, which is also the case for other endocrine tumors [2, 16]. Correlations between Ki67 expression and malignant behavior have been reported in other adrenal gland primary neoplasms such as pheochromocytoma and adult adrenocortical tumors [20, 25-31]. In pediatric adrenocortical tumors, some studies have shown a significant correlation between the Wieneke score and Ki67, with a $15 \%$ cutoff being independently associated with prognosis [14, 21]. In our study, a Ki67 proliferation index $<15 \%$ essentially ruled out malignancy, as all patients were progression free in this group. Furthermore, this index was significantly higher in patients with unfavorable outcomes.

Regarding clinical and biological data, the data for our cohort are consistent with what was found in previous series, supporting the scientific validation of this study and the accuracy of the results. We confirmed a female predominance, mostly in the $>10$-year-old group, which is the subgroup that also usually displays adverse outcomes. Indeed, age $>10$ years and $\leq 4$ years at diagnosis was previously shown to have a significant difference regarding OS and PFS [7, 32]. Almost 50\% of patients in our series were aged $\leq 4$ years, which was strongly associated with favorable outcomes, confirming the distinct entity that characterized this subgroup of patients. As previously reported, about $90 \%$ of tumors were functional, and virilization (alone or in combination with other signs of overproduction) was the most common presenting syndrome. In contrast to results from other series, no single endocrinological clinical feature correlated with a more favorable or unfavorable prognosis $[2,7,8]$. However, the major prognostic factors that we did identify are consistent with those previously reported, namely lymph node involvement, metastatic spread, and incomplete resection (micro- or macroscopic residual tumor or tumor spillage) [2, 7]. About $75 \%$ of patients had stage I or II disease, whereas only $5 \%$ of patients had stage IV in our study. Because disease stage correlates greatly with disease outcome (in univariate and multivariate analyses), this explains the high 5-year eventfree and OS estimates that were up to $80 \%$ for the whole cohort. This figure is much higher in our series, compared with others, due to the inclusion of all adrenocortical tumors independent of initial diagnosis [2, 7, 33]. As tumors were initially unselected and inclusion was comprehensive based on various databases from 23 French hospitals, this demonstrates that pediatric adrenocortical tumors are overall benign neoplasms confined to the adrenal gland [16].

In pediatric adrenocortical tumors, the treatment recommendations defined by the Children's Oncology Group are based quasi-exclusively on tumor stage as defined by the International Pediatric Adrenocortical Tumor Registry. In all studies that defined the major prognostic factors of these tumors, the value of histologic features was not assessed due to "the difficulty of consistently discriminating benign (adenomas) from malignant (carcinomas) neoplasms" [2]. Currently, treatment algorithms are clear for stage I and stage IV disease (i.e., surgery alone and perioperative 


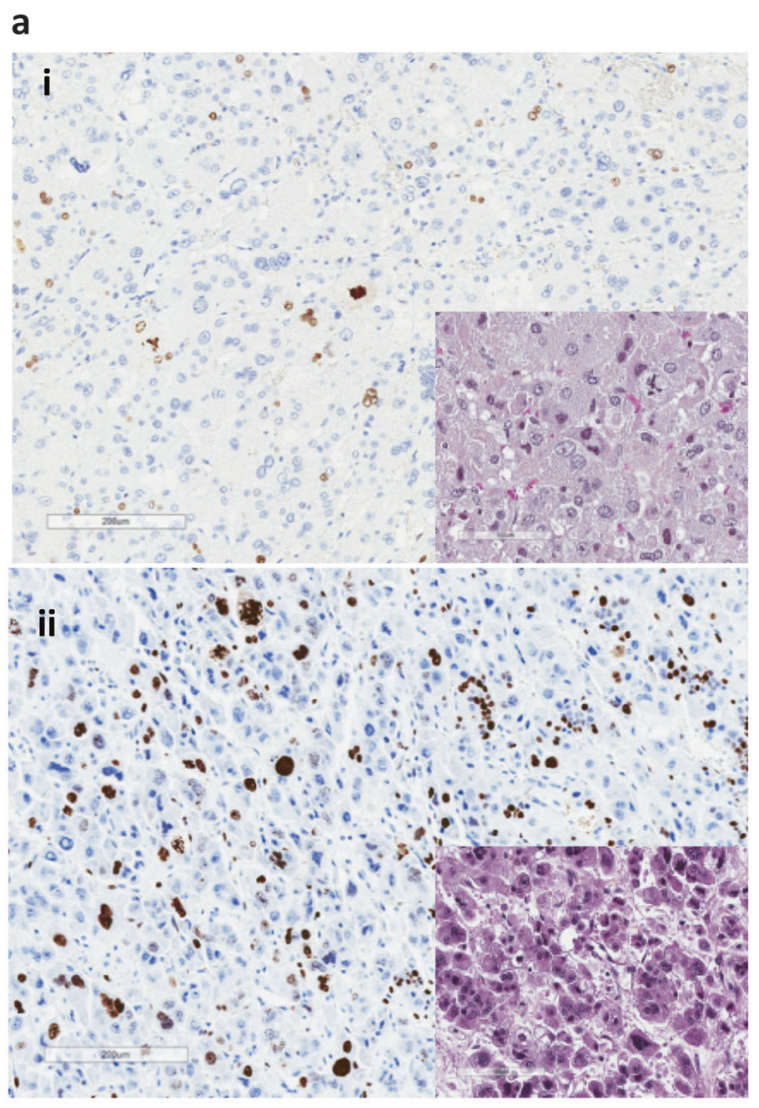

b

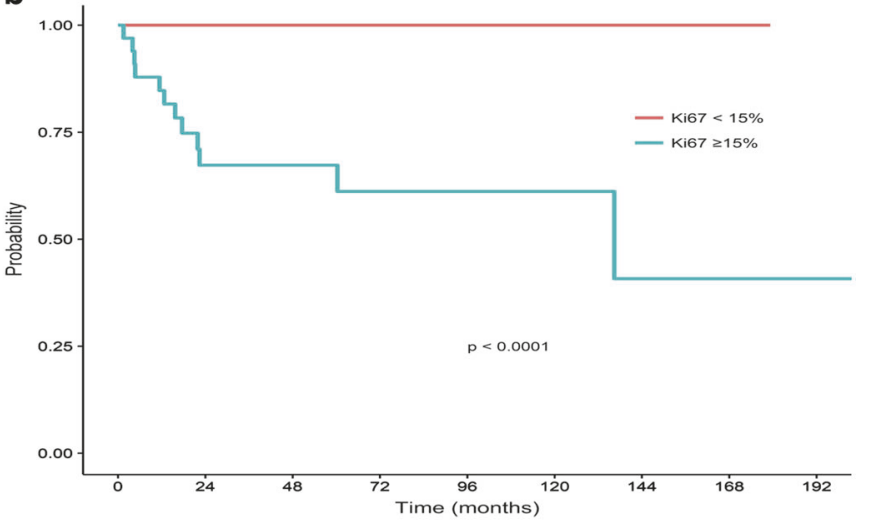

Number at risk

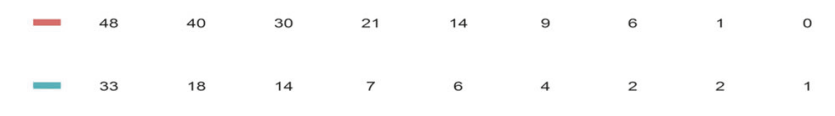

C

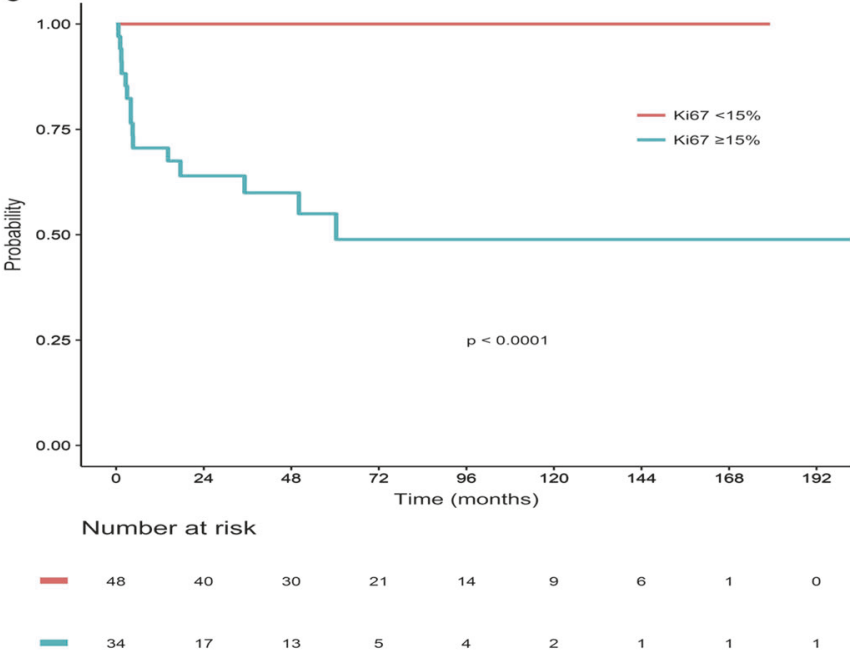

index $<15 \%$ and (ii) tumor with a Ki67 labeling index $\geq 15 \%$. Estimated overall survival (OS) (b) and progression-free survival (PFS) (c) for the whole cohort of patients as they relate to Ki67 index

and to assess the role of a five-item score integrating adrenal capsular invasion, venous invasion, confluent necrosis, mitotic count $>15 / 20$ high-power fields, and Ki67 $\geq 15 \%$, for the whole cohort and for stages II-III tumors. Regardless of tumor stage, no deaths occurred in this series when the tumor possessed less than two criteria. Furthermore, a score $>2$ was independently associated with a worse outcomes in multivariate analyses. All stage II tumors with a microscopic score $\leq 2$ had favorable outcomes whereas some of them were classified as "intermediate" $(n=1)$ or "malignant" $(n=3)$ with the Wieneke score. Among stage II patients with more than two unfavorable microscopic features, all were in complete remission at the end of follow-up (except for one non-tumor-related death), including two with tumor progression that were treated with complete surgery and systemic therapy. The outcomes for 


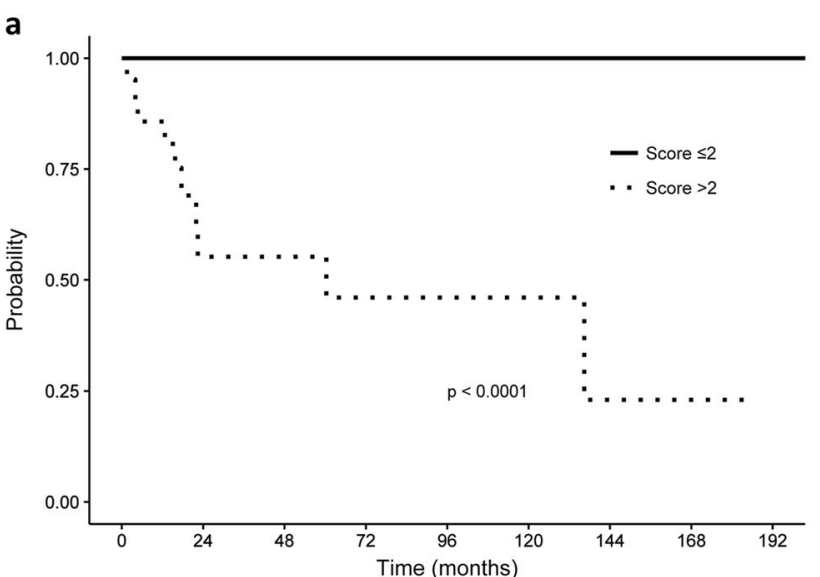

Number at risk

$\begin{array}{lccccccccc}\text { Score } \leq 2 & 58 & 50 & 37 & 24 & 16 & 11 & 7 & 2 & 1 \\ \text { Score }>2 & 21 & 8 & 7 & 4 & 4 & 2 & 1 & 1 & 0\end{array}$

Fig. 5 Estimated overall survival (OS) (a) and progression-free survival (PFS) (b) for the whole cohort of patients using proposed microscopic score including four prognostic microscopic features

stage II patients with up to two malignant histologic features is still unpredictable, as some relapsed and some were in complete remission after a long-term follow-up without any adjuvant therapy. It is clear that, regardless of microscopic features, localized totally resected tumors with high tumor volume have a relatively favorable outcome compared with stages III and IV tumors. However, when other unfavorable prognostic factors (age $>10$ years old, for example) are present, less than two malignant microscopic features could reassure clinicians regarding disease evolution.

Despite the large size of this cohort, we acknowledge that the major limitation of this study is the insufficient number of patients included, especially regarding our subgroup analyses. This is highlighted by some of the HRs that showed wide $95 \%$ CIs in our study. However, as the outcomes are globally well known for stages I and IV tumors, it seems important to focus on localized bulky tumors and on stage III disease with few poor prognostic factors, which are currently the most unpredictable and challenging tumors for both clinicians and pathologists to prognosticate. The retrospective nature of the data collection with a 5-year median follow-up allowed us to determine the prognostic value of numerous parameters since the majority of events occurred in the first 2 years following diagnosis. Regarding the two cases with only biopsy specimens available due to inoperable tumors, only a Wieneke score estimation was possible. Inclusion of these patients, although perhaps questionable, permits for better overall stage representation of the cohort since they belong to the small group of patients who initially presented with advanced-stage disease (stages III and IV).

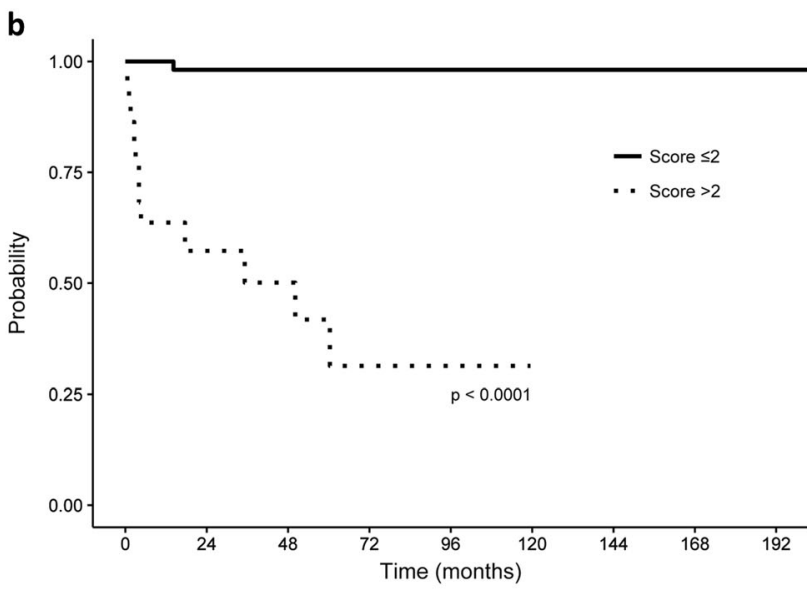

Number at risk

$\begin{array}{lccccccccc}\text { Score } \leq 2 & 58 & 49 & 37 & 24 & 16 & 11 & 7 & 2 & 1 \\ \text { Score }>2 & 22 & 8 & 6 & 2 & 2 & 0 & 0 & 0 & 0\end{array}$

(adrenal capsular invasion, venous invasion, confluent necrosis, mitotic count $>15 / 20$ high-power fields) and Ki67 labeling index $\geq$ $15 \%$

The evidence from our current study highlights that while the Wieneke score includes major prognostic criteria, it remains inadequate, as currently defined, in predicting disease outcomes and guiding treatment recommendations, especially for scores $>3$. We propose to refine the purpose and role of the pathological examination in the prognostic assessment of pediatric adrenocortical tumors, using a two-step approach as illustrated in Fig. 6. First, for non-metastatic disease, the primary role of pathological staging is the assessment of satisfactory or unsatisfactory surgical resection, differentiating stages I/II from stage III disease. This implies a close collaboration between radiologists, pathologists, and surgeons, as well as a rigorous gross examination of the surgical specimen with inked margins. For completely resected tumors, a tumor weight of up to $200 \mathrm{~g}$ should be reported to confirm stage II disease. In the case of weight overestimation due to associated adjacent organ resection or in the case of fragmented ruptured tumor, tumor volume could be estimated from tumor measurements obtained from imaging. We believe that group III staging should be considered if inflammatory changes suggestive of prior tumor rupture are present microscopically. Finally, when the surgical resection specimen includes lymph node dissection, the involvement of retroperitoneal lymph nodes is assessed by the pathologist and defines stage III disease. After a proper Children's Oncology Group tumor staging assessment by the pathologist, which appears to be the most reliable determinant of prognosis, the use of a consistent and simple microscopic scoring system including Ki67 proliferation index could be of interest to 


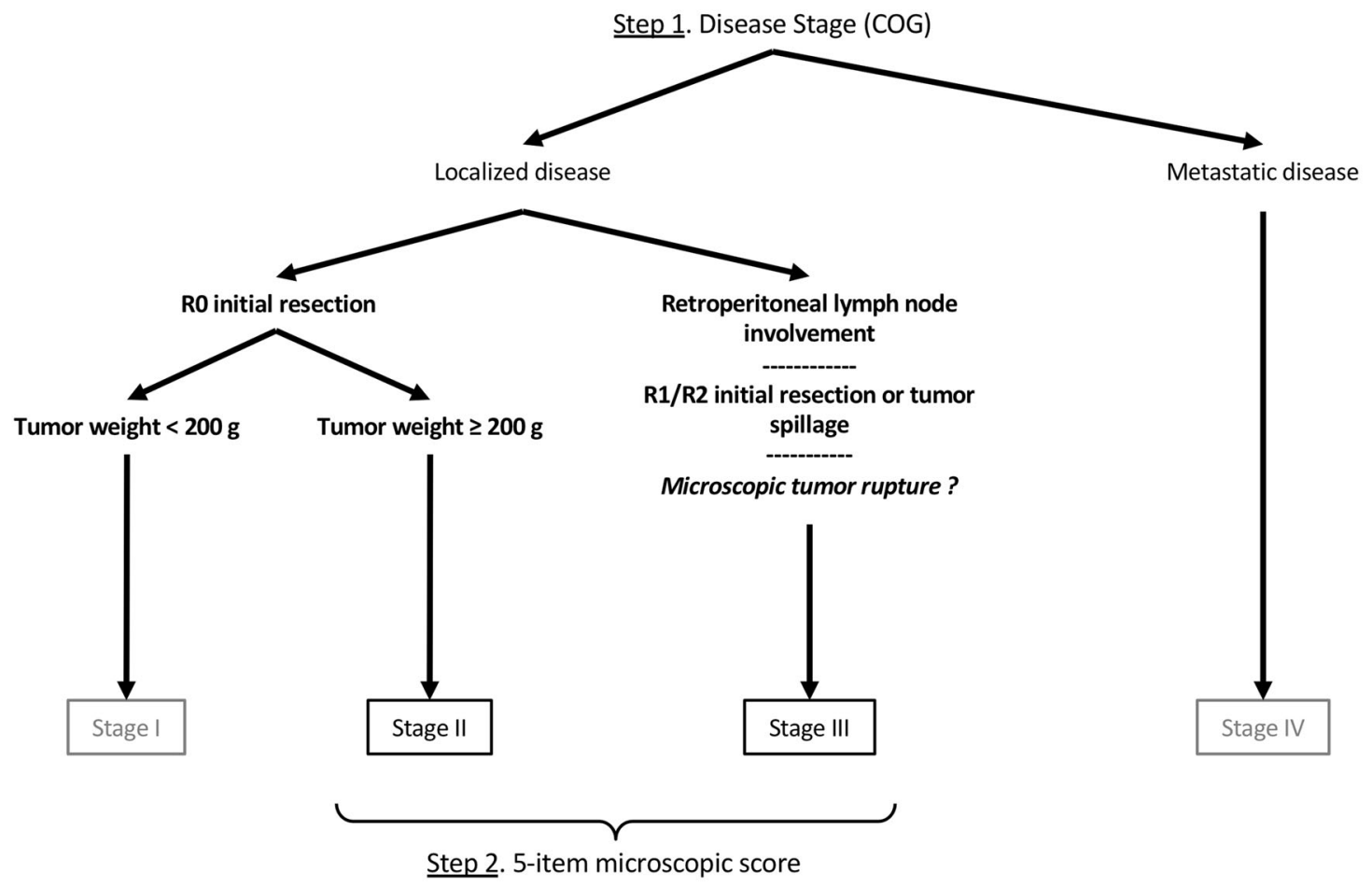

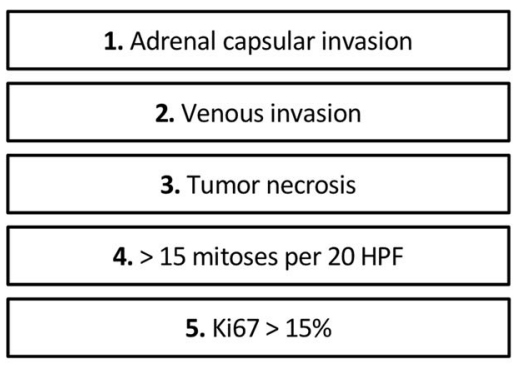

Fig. 6 Proposal of a two-step pathological approach in localized stages II and III pediatric adrenal cortical neoplasms to consider need of adjuvant systemic therapy. In step 1, items in bold are supplied or confirmed by pathological study. R0: microscopically complete initial

stratify tumors with favorable vs. unfavorable histology, especially for localized disease (Fig. 6). Given the rarity of these tumors, international cooperation is fundamental to collect more complete data and to validate these findings especially for intermediate groups (stages II-III). For stages II and III tumors displaying few poor prognostic factors, we propose that unfavorable vs. favorable tumor histology criteria should be assigned, based on whether or not up to two of the following malignancy-related microscopic features are present: adrenal capsular invasion, venous invasion, confluent tumor necrosis, mitotic count $>15 / 20$ high-power fields, and Ki67 $\geq 15 \%$ (Fig. 6). Using this algorithm, oncologists may decide against recommending adjuvant therapy for patients with favorable histology tumors, considering $\leq 2$ features: "favorable histology tumor"

$>2$ features: "unfavorable histology tumor"

I

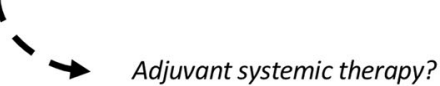

surgical resection; R1: initial surgical resection with microscopic residuals or tumor spillage; R2: initial surgical resection with macroscopic residuals

this group's lower risk of disease progression and the toxic side effect profile and overall minimal benefit of these therapies.

Acknowledgements We thank "Enfants, cancers et Santé" and the Hospices Civils de Lyon for their financial support. The association "Enfants, cancers et Santé" does not participate in any of the analyses of this manuscript. In addition, we thank all pathologists who sent tumor specimens, as well as the pediatric oncologists, surgeons, and endocrinologists who collaborated in sending clinical data about the cases in this study. We would also like to thank Jessica Chan and Ashley Flaman for their help in reviewing the manuscript.

\section{Compliance with ethical standards}

Conflict of interest The authors declare that they have no conflict of interest. 


\section{References}

1. Ribeiro RC, Pinto EM, Zambetti GP, et al. The International Pediatric Adrenocortical Tumor Registry initiative: contributions to clinical, biological, and treatment advances in pediatric adrenocortical tumors. Mol Cell Endocrinol. 2012;351:37-43.

2. Michalkiewicz E, Sandrini R, Figueiredo B, et al. Clinical and outcome characteristics of children with adrenocortical tumors: a report from the International Pediatric Adrenocortical Tumor Registry. J Clin Oncol. 2004;22:838-45.

3. Bilimoria KY, Shen WT, Elaraj D, et al. Adrenocortical carcinoma in the United States: treatment utilization and prognostic factors. Cancer. 2008;113:3130-6.

4. Pinto EM, Billerbeck AEC, Villares MCBF, et al. Founder effect for the highly prevalent R337H mutation of tumor suppressor p53 in Brazilian patients with adrenocortical tumors. Arq Bras Endocrinol Metabol. 2004;48:647-50.

5. Ribeiro RC, Sandrini F, Figueiredo B, et al. An inherited p53 mutation that contributes in a tissue-specific manner to pediatric adrenal cortical carcinoma. Proc Natl Acad Sci USA. 2001;98:9330-5.

6. Lalli E, Figueiredo BC. Pediatric adrenocortical tumors: what they can tell us on adrenal development and comparison with adult adrenal tumors. Front Endocrinol. 2015;6:23.

7. Cecchetto G, Ganarin A, Bien E, et al. Outcome and prognostic factors in high-risk childhood adrenocortical carcinomas: a report from the European Cooperative Study Group on Pediatric Rare Tumors (EXPeRT). Pediatr Blood Cancer. 2017;64:e26368.

8. Sandrini R, Ribeiro RC, DeLacerda L. Childhood adrenocortical tumors. J Clin Endocrinol Metab. 1997;82:2027-31.

9. Ribeiro RC, Figueiredo B. Childhood adrenocortical tumours. Eur J Cancer Oxf Engl 1990. 2004;40:1117-26.

10. Weiss LM. Comparative histologic study of 43 metastasizing and nonmetastasizing adrenocortical tumors. Am J Surg Pathol. 1984;8:163-9.

11. Weiss LM, Medeiros LJ, Vickery AL. Pathologic features of prognostic significance in adrenocortical carcinoma. Am J Surg Pathol. 1989;13:202-6.

12. Wieneke JA, Thompson LDR, Heffess CS. Adrenal cortical neoplasms in the pediatric population: a clinicopathologic and immunophenotypic analysis of 83 patients. Am J Surg Pathol. 2003;27:867-81.

13. Sbragia L, Oliveira-Filho AG, Vassallo J, et al. Adrenocortical tumors in Brazilian children: immunohistochemical markers and prognostic factors. Arch Pathol Lab Med. 2005;129:1127-31.

14. Das S, Sengupta M, Islam N, et al. Weineke criteria, Ki-67 index and p53 status to study pediatric adrenocortical tumors: is there a correlation? J Pediatr Surg. 2016;51:1795-1800.

15. Magro G, Esposito G, Cecchetto G, et al. Pediatric adrenocortical tumors: morphological diagnostic criteria and immunohistochemical expression of matrix metalloproteinase type 2 and human leucocyte-associated antigen (HLA) class II antigens. Results from the Italian Pediatric Rare Tumor (TREP) Study project. Hum Pathol. 2012;43:31-39.

16. Dehner LP, Hill DA. Adrenal cortical neoplasms in children: why so many carcinomas and yet so many survivors? Pediatr Dev Pathol. 2009;12:284-91.
17. Tucci S, Martins ACP, Suaid HJ, et al. The impact of tumor stage on prognosis in children with adrenocortical carcinoma. J Urol. 2005; 174:2338-42.

18. Ru W, Yang M, Xu S, et al. Management and prognosis of adrenocortical tumors in children: can we find out an appropriate points-scoring system to predict prognosis? Pediatr Surg Int. 2017;33:705-11.

19. Chatterjee G, DasGupta S, Mukherjee G, et al. Usefulness of Wieneke criteria in assessing morphologic characteristics of adrenocortical tumors in children. Pediatr Surg Int. 2015;31:563-71.

20. Beuschlein F, Weigel J, Saeger W, et al. Major prognostic role of Ki67 in localized adrenocortical carcinoma after complete resection. J Clin Endocrinol Metab. 2015;100:841-9.

21. Pinto EM, Rodriguez-Galindo C, Pounds SB, et al. Identification of clinical and biologic correlates associated with outcome in children with adrenocortical tumors without germline TP53 mutations: a St Jude Adrenocortical Tumor Registry and Children's Oncology Group Study. J Clin Oncol. 2017; 35:3956-63.

22. Cohen J. Weighted kappa: nominal scale agreement provision for scaled disagreement or partial credit. Psychol Bull. 1968;70:213.

23. Firth D. Bias reduction of maximum likelihood estimates. Biometrika. 1993;80:27-38.

24. Cagle PT, Hough AJ, Pysher TJ, et al. Comparison of adrenal cortical tumors in children and adults. Cancer. 1986;57:2235-7.

25. Brown HM, Komorowski RA, Wilson SD, et al. Predicting metastasis of pheochromocytomas using DNA flow cytometry and immunohistochemical markers of cell proliferation. Cancer. 1999;86:1583-9.

26. Gupta D, Shidham V, Holden J, et al. Prognostic value of immunohistochemical expression of topoisomerase alpha II, MIB$1, \mathrm{p} 53$, E-cadherin, retinoblastoma gene protein product, and HER-2/neu in adrenal and extra-adrenal pheochromocytomas. Appl Immunohistochem Mol Morphol. 2000;8:267.

27. August C, August K, Schroeder S, et al. CGH and CD 44/MIB-1 immunohistochemistry are helpful to distinguish metastasized from nonmetastasized sporadic pheochromocytomas. Mod Pathol. 2004;17:1119-28.

28. Erickson LA. Challenges in surgical pathology of adrenocortical tumours. Histopathology. 2018;72:82-96.

29. Wachenfeld C, Beuschlein F, Zwermann O, et al. Discerning malignancy in adrenocortical tumors: are molecular markers useful? Eur J Endocrinol. 2001;145:335-41.

30. Duregon E, Molinaro L, Volante M, et al. Comparative diagnostic and prognostic performances of the hematoxylin-eosin and phospho-histone $\mathrm{H} 3$ mitotic count and $\mathrm{Ki}-67$ index in adrenocortical carcinoma. Mod Pathol. 2014;27:1246-54.

31. Fassnacht M, Kroiss M, Allolio B. Update in adrenocortical carcinoma. J Clin Endocrinol Metab. 2013;98:4551-64.

32. Dall'Igna P, Virgone C, De Salvo GL, et al. Adrenocortical tumors in Italian children: analysis of clinical characteristics and P53 status. Data from the national registries. J Pediatr Surg. 2014;49:1367-71.

33. Leblond P, Delebarre M, Aubert S. Management of adrenocortical carcinomas in children. Bull Cancer (Paris). 2011;98:595-605. 